\title{
Biological functions and clinical significance of the newly identified long non-coding RNA RP1-85F18.6 in colorectal cancer
}

\author{
YESHUO MA ${ }^{1}$, YIFEI CHEN ${ }^{2}$, CHANGWEI LIN ${ }^{3}$ and GUI HU ${ }^{3}$ \\ ${ }^{1}$ Department of Cardiology, The Third Xiangya Hospital of Central South University; \\ ${ }^{2}$ Department of Otolaryngology-Head Neck Surgery, The Fourth Hospital of Changsha \\ (The Changsha Affiliated Hospital of Hunan Normal University), Hunan Normal University; \\ ${ }^{3}$ Department of Gastrointestinal Surgery, The Third Xiangya Hospital of Central South University, \\ Changsha, Hunan 410013, P.R. China
}

Received March 31, 2018; Accepted September 7, 2018

DOI: 10.3892/or.2018.6694

\begin{abstract}
The biological functions of long non-coding RNAs (lncRNAs) in cancer have not been fully elucidated. The present study demonstrated that the expression of a newly identified lncRNA, RP1-85F18.6, was upregulated in colorectal cancer (CRC) tissues and cell lines. Knockdown of lncRNA RP1-85F18.6 served a key role in tumor inhibition, reduced cell proliferation and invasion, disrupted the cell cycle, and increased apoptosis and pyroptosis of CRC cells. Conversely, overexpression of 1ncRNA RP1-85F18.6 exerted the opposite effects. Furthermore, silencing lncRNA RP1-85F18.6 decreased $\Delta \mathrm{Np} 63$ expression at both the mRNA and protein levels. Furthermore, co-transfection with $\triangle N p 63$ siRNA and 1ncRNA RP1-85F18.6-expressing vector attenuated the tumor-promoting effects of IncRNA RP1-85F18.6 overexpression. The expression levels of lncRNA RP1-85F18.6, $\Delta$ Np63 and gasdermin D (GSDMD) were revealed to be associated with lymph node and distant metastases in patients with CRC, and therefore may serve as predictors in CRC. The findings of the present study suggested that lncRNA RP1-85F18.6 may trigger $\mathrm{CRC}$ cell proliferation, invasion and cell cycle disrup-
\end{abstract}

Correspondence to: Professor Gui Hu, Department of Gastrointestinal Surgery, The Third Xiangya Hospital of Central South University, 138 Tongzipo Road, Changsha, Hunan 410013, P.R. China E-mail: hugui22@qq.com

Abbreviations: lncRNA, long non-coding RNA; CRC, colorectal cancer; GSDMD, gasdermin D; ncRNA, non-protein-coding ribonucleic acid; NCT, non-cancerous tissue; RT-qPCR, reverse transcription-quantitative polymerase chain reaction; $\mathrm{LDH}$, lactate dehydrogenase; ROC curve, receiver operating characteristic curve; AUC, area under the ROC curve; CEA, carcinoembryonic antigen

Key words: long non-coding RNA, $\Delta \mathrm{Np} 63$, colorectal cancer, pyroptosis, apoptosis, gasdermin D tion, and suppress apoptosis and pyroptosis of CRC cells through regulating $\triangle \mathrm{Np} 63$ expression. Therefore, lncRNA RP1-85F18.6 and $\Delta$ Np63 may be considered unfavorable biomarkers, whereas GSDMD may be a favorable biomarker in CRC; these markers may prove valuable in the future diagnosis and prognosis of CRC.

\section{Introduction}

Colorectal cancer (CRC) is the second most prevalent type of cancer and the third leading cause of cancer-associated mortality worldwide (1). Despite significant advances in the diagnosis and treatment of CRC, the overall survival rate of CRC remains unsatisfactory $(2,3)$. Therefore, it is necessary to identify the molecular mechanisms underlying the occurrence and development of CRC, in order to develop more effective diagnostic and therapeutic methods.

Genomic studies have confirmed that only $2 \%$ of human gene transcripts encode proteins, whereas numerous transcripts encode non-coding ribonucleic acids (ncRNAs) (4). Long ncRNAs (lncRNAs) are a subtype of ncRNAs $>200$ nucleotides long, which lack protein-coding ability. Although the exact functions of IncRNAs remain unclear, they appear to regulate numerous biological behaviors through epigenetic regulation, transcription and post-transcriptional processing (5). Accumulating evidence has indicated that the aberrant expression of lncRNAs may regulate cancer cell proliferation, migration, invasion, apoptosis and metastasis $(6,7)$.

p63 is a member of the p53 family, which is highly homologous and structurally similar to p53 (8).p63 has two isoforms, TAp63 and $\Delta \mathrm{Np} 63$, which differ at the N-terminal. TAp63 contains an N-terminal transactivation domain, whereas $\Delta$ Np63 lacks this domain (9). These two isoforms perform different functions during tumorigenesis. TAp63 acts as a tumor suppressor, similar to p53, and induces cell cycle arrest and cell apoptosis (10). Conversely, $\Delta$ Np63 acts as an oncogene, facilitating proliferation, survival, invasion, metastasis and chemoresistance, and reducing apoptosis of various cancer 
cells (11-13). Numerous studies have revealed that lncRNAs mediate the biological functions of CRC cells through modulating p53 expression (14-16). However, the number of studies on lncRNAs regulating CRC tumorigenesis by targeting p63 is limited. The present study aimed to determine whether there is a newly identified lncRNA targeting $\Delta N p 63$, and to investigate the biological functions of this newly identified lncRNA in the proliferation, cell cycle progression, invasion, apoptosis and pyroptosis of CRC cells, in the aim of providing a novel target for the treatment of patients with CRC.

\section{Materials and methods}

Ethics statement. The present study was approved by the Ethics Committee of The Third Xiangya Hospital of Central South University (Changsha, China). The study protocol conformed to the principles outlined in the Helsinki Declaration and to local legislation. Informed consent was obtained from all of the participants.

Patient specimens. A total of 34 pairs of human primary CRC tissues and corresponding non-cancerous tissues (NCTs) were collected from patients who had undergone tumor resection at the Department of Gastrointestinal Surgery, The Third Xiangya Hospital of Central South University between October 2014 and June 2015. The specimens were immediately frozen in liquid nitrogen following surgical resection and maintained at $-80^{\circ} \mathrm{C}$. The clinical characteristics of all patients, including age, tumor site, tumor differentiation, stage, lymph node metastasis and distant metastasis, are summarized in Table I. Cancer staging was performed according to the 7th TNM classification by the American Joint Committee on Cancer (17). None of the patients underwent radiotherapy or chemotherapy prior to surgery.

RNA isolation and reverse transcription-quantitative polymerase chain reaction $(R T-q P C R)$ analysis. RNA was extracted from CRC tissues/NCTs, or fresh cultured cells using TRIzol ${ }^{\circledR}$ reagent (Invitrogen; Thermo Fisher Scientific, Inc., Waltham, MA, USA) according to the manufacturer's protocol. RT of $3 \mu \mathrm{g}$ total RNA was performed using RevertAid Reverse Transcriptase (Thermo Fisher Scientific, Inc.), according to the manufacturer's protocol. RT-qPCR was performed using the SYBR-Green Master Mix (Bio-Rad Laboratories, Inc., Hercules, CA, USA) and was run using a thermal cycler (Bio-Rad Laboratories, Inc.). The thermal cycling conditions were as follows: $95^{\circ} \mathrm{C}$ for $2 \mathrm{~min}$, followed by 40 cycles at $95^{\circ} \mathrm{C}$ for $30 \mathrm{sec}, 50^{\circ} \mathrm{C}$ for $30 \mathrm{sec}$ and $72^{\circ} \mathrm{C}$ for $30 \mathrm{sec}$, and a final extension step at $72^{\circ} \mathrm{C}$ for $10 \mathrm{~min}$. GAPDH was used for normalization. Relative gene expression was calculated using the $2^{-\Delta \Delta \mathrm{Cq}}$ method (18). The primers used in the present study are listed in Table II.

Cell culture. The NCM460 human colorectal epithelial cell line, and the SW480, SW620 and HCT116 CRC cell lines were obtained from Nanjing KeyGen Biotech Co., Ltd. (Nanjing, China). The cells were cultured in Dulbecco's modified Eagle's medium containing $10 \%$ fetal bovine serum (FBS) (HyClone; GE Healthcare, Logan, UT, USA) at $37^{\circ} \mathrm{C}$ in a humidified atmosphere containing $5 \% \mathrm{CO}_{2}$.
Cell transfection. LncRNA RP1-85F18.6 small interfering (si)RNA, $\triangle \mathrm{Np} 63$ siRNA and negative control (NC) siRNA were purchased from Guangzhou RiboBio Co., Ltd. (Guangzhou, China). The sequences were as follows: lncRNA RP1-85F18.6 siRNA, 5'-GACTCCGCCGTGAACCCT TCA-3'; $\triangle$ Np63 siRNA, 5'-ACAAUGCCCAGACUCAAU UUU-3'. A scramble siRNA (siN05815122147) was used as the NC siRNA. Once the SW620 cells reached $70 \%$ confluence, the cells were transfected for $48 \mathrm{~h}$ at $37^{\circ} \mathrm{C}$ with lncRNA RP1-85F18.6 siRNA, $\triangle$ Np63 siRNA or NC siRNA using Lipofectamine ${ }^{\circledR} 2000$ (Invitrogen; Thermo Fisher Scientific, Inc.), according to the manufacturer's protocol. The siRNAs were diluted to a final concentration of $60 \mathrm{nM}$ for transfection. In addition, the entire sequence of human lncRNA RP1-85F18.6 was amplified from SW620 cells using PCR and cloned into the pcDNA3.1 vector. The negative control empty vector, which was purchased from Shanghai GeneChem Co., Ltd. (Shanghai, China), and the lncRNA RP1-85F18.6 plasmid were transfected into SW620 cells using Lipofectamine ${ }^{\circledR} 2000$ (Invitrogen; Thermo Fisher Scientific, Inc.), according to the manufacturer's protocol. The plasmid was diluted to a final concentration of $2 \mu \mathrm{g} / \mathrm{ml}$ for transfection.

Cell proliferation assay. Transfected SW620 cells were cultured in 96-well plates and incubated for 24, 48 and $72 \mathrm{~h}$. Optical density values were measured using the MTT Cell Proliferation and Cytotoxicity Assay kit (Beyotime Institute of Biotechnology, Shanghai, China), according to the manufacturer's protocol. Cell proliferation was calculated using the absorbance values, which were measured at $490 \mathrm{~nm}$ at each time point.

Flow cytometric analysis of apoptosis. Cell apoptosis was estimated using flow cytometric analysis with the Apoptosis Detection kit (Nanjing KeyGen BioTech Co., Ltd.), according to the manufacturer's protocol. After transfection for $48 \mathrm{~h}$, the cells were washed with ice-cold PBS and resuspended with binding buffer. Subsequently, the cells were incubated with propidium iodide (PI) and Annexin $\mathrm{V}$ at room temperature for $15 \mathrm{~min}$ in the dark. The cells were then resuspended with PBS and analyzed by flow cytometry (BD Biosciences, Franklin Lakes, NJ, USA) and FlowJo software (v7.6.2, FlowJo; LLC, Ashland, OR, USA).

Cell cycle analysis. Cell cycle analysis was performed as previously described (19). Briefly, after transfection for $48 \mathrm{~h}$, the cells were washed with PBS and fixed with ice-cold $70 \%$ ethanol at $4^{\circ} \mathrm{C}$ overnight. Fixed cells were washed with PBS and incubated with PI and RNase, which were obtained from the Cell Cycle Detection kit (Nanjing KeyGen BioTech Co., Ltd.), for 30 min at room temperature in the dark. Subsequently, the incubated cells were analyzed by flow cytometry (BD Biosciences) and FlowJo software (v7.6.2, FlowJo; LLC).

Transwell assay. Cellular invasion was evaluated using Transwell migration chambers precoated with a layer of Matrige ${ }^{\circledR}$. This assay was performed as previously described at $48 \mathrm{~h}$ post-transfection (20). Briefly, cells suspended in $100 \mu \mathrm{l}$ medium without FBS were seeded in the upper chamber $\left(10^{4}\right.$ cells/well). To the lower chamber, $600 \mu 1$ medium 


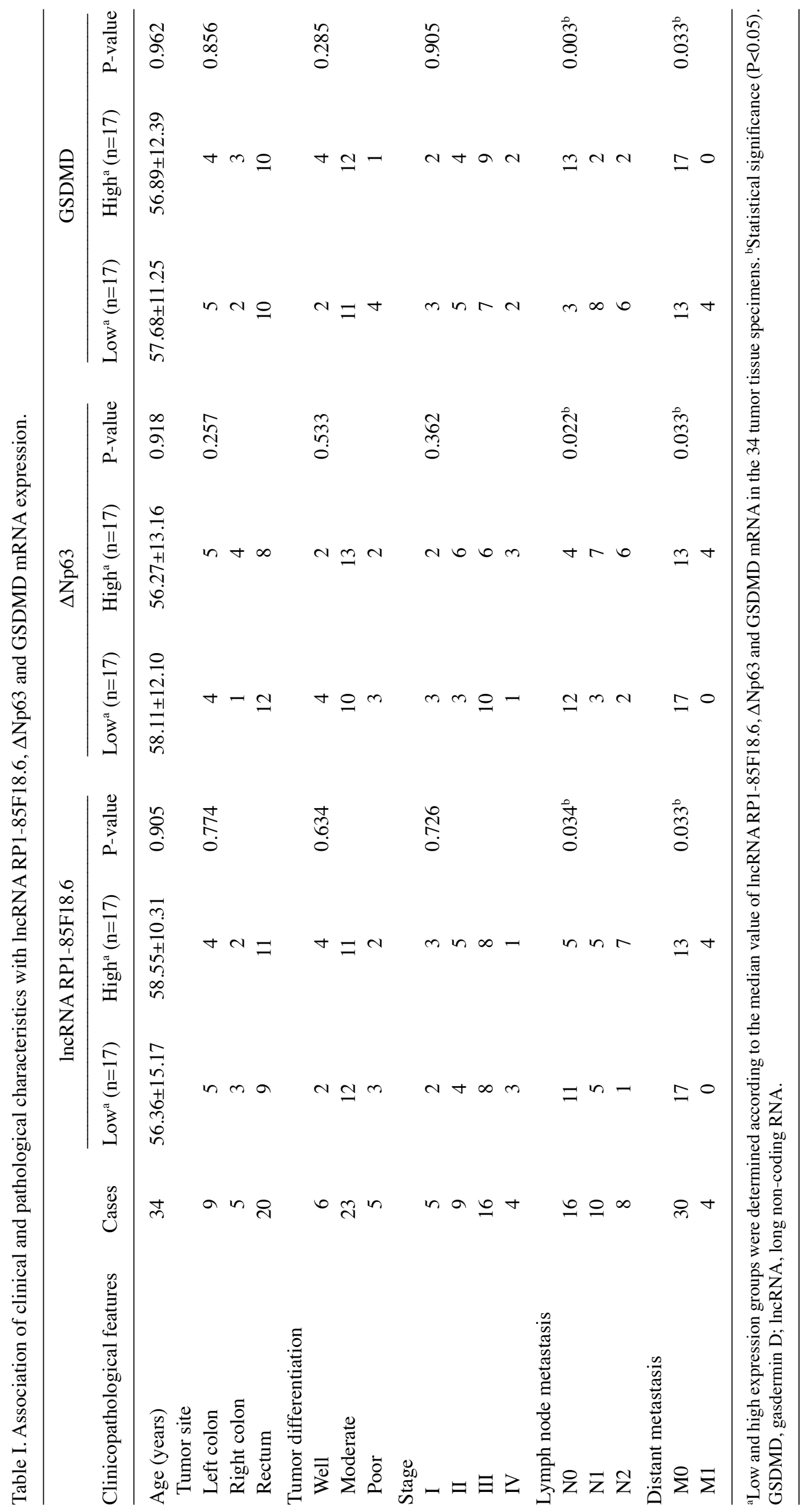


Table II. Reverse transcription-quantitative polymerase chain reaction primers used in this study.

\begin{tabular}{ll}
\hline Gene name & \multicolumn{1}{c}{ Primer sequence (5'-3') } \\
\hline $\begin{array}{ll}\text { lncRNA } \\
\text { RP1-85F18.6 }\end{array}$ & Forward: GGCTCTTTGCTCACATCG \\
& Reverse: AAGGAAACCACAGGCTCA \\
$\Delta$ Np63 & Forward: GAAGAAAGGACAGCAGCAT \\
& TGA \\
& Reverse: GGGACTGGTGGACGAGGAG \\
TAp63 & Forward: TGTATCCGCATGCAGGACT \\
& Reverse: CTGTGTTATAGGGACTGGTG \\
& GAC \\
GSDMD & Forward: GTGTGTCAACCTGTCTATCA \\
& AGG \\
& Reverse: CATGGCATCGTAGAAGTGGAAG \\
GAPDH & Forward: ACCACAGTCCATGCCATCAC \\
& Reverse: TCCACCACCCTGTTGCTGTA \\
\hline
\end{tabular}

GSDMD, gasdermin D; lncRNA, long non-coding RNA.
Lactate dehydrogenase $(\mathrm{LDH})$ release assay. After transfection for $48 \mathrm{~h}$, the supernatants of transfected cells were collected to measure LDH release using LDH Cytotoxicity Assay kit, according to the manufacturer's protocol (Beyotime Institute of Biotechnology). Data were collected based on the absorbance at $490 \mathrm{~nm}$, which was measured using a microplate reader (Thermo Fisher Scientific, Inc.).

Statistical analysis. All experiments were repeated three times and data are expressed as the means \pm standard deviation. All statistical calculations were performed using GraphPad Prism version 6.01 (GraphPad Software, Inc., La Jolla, CA, USA) and SPSS (PASW Statistics) version 20 (IBM Corporation, Armonk, NY, USA). The Mann-Whitney U test was used to compare differences between two groups. One-way analysis of variance followed by the Student-Newman-Keul's test was used to compare multiple groups. The correlation between two factors was determined using the Spearman's rank correlation test. The sensitivity and specificity in CRC tissues were calculated using the Youden's index. The area under the receiver operating characteristic (ROC) curve (AUC) was also estimated. $\mathrm{P}<0.05$ was considered to indicate a statistically significant difference.

\section{Results}

supplemented with $20 \%$ FBS was added. After $24 \mathrm{~h}$ incubation at $37^{\circ} \mathrm{C}$, cells on the top of the membrane were removed with cotton swabs. The cells that invaded through the membrane were washed with PBS, fixed in $4 \%$ methanol for $20 \mathrm{~min}$ and stained with $0.1 \%$ crystal violet for $10 \mathrm{~min}$ at room temperature. The number of invasive cells was counted in five randomly selected fields under a light microscope (Leica Microsystems GmbH, Wetzlar, Germany).

Protein extraction and western blotting. Protein extraction and western blotting were performed as previously described (21). Briefly, proteins were extracted from tissues and cells using radioimmunoprecipitation assay lysis buffer containing $1 \% 100 \mathrm{mM}$ phenylmethylsulfonyl fluoride (both from Beyotime Institute of Biotechnology). Protein concentration was examined using the bicinchoninic acid method (Nanjing KeyGen Biotech Co., Ltd.). Subsequently, proteins $(20 \mu \mathrm{g})$ were separated by $10 \%$ SDS-PAGE and were transferred to polyvinylidene fluoride membranes. The membranes were blocked in 5\% non-fat milk for $2 \mathrm{~h}$ at room temperature, and were incubated with the following primary antibodies at $4^{\circ} \mathrm{C}$ overnight: Anti- $\beta$-actin (1:2,000, cat. no. 20536-1-AP) and anti-gasdermin D (GSDMD; 1:1,000, cat. no. 20770-1-AP) (both from Wuhan Sanying Biotechnology, Wuhan, China); anti- $\Delta$ Np63 (1:500, cat. no. 619001) and anti-TAp63 (1:500, cat. no. 618901) (both from BioLegend, Inc., San Diego, CA, USA). After washing with PBS-0.1\% Tween, the membranes were incubated with anti-rabbit immunoglobulin $\mathrm{G}$ secondary antibody (1:5,000, cat. no. SA00001-2; Wuhan Sanying Biotechnology) for $1 \mathrm{~h}$ at room temperature. The images were obtained using Advansta WesternBright enhanced chemiluminescence (Advansta lnc., Menlo Park, CA, USA) and the ChemiDoc $^{\mathrm{TM}}$ MP Imaging system (Bio-Rad Laboratories, Inc.). p63-associated IncRNA identification. To identify novel lncRNAs targeting p63, a microarray analysis was performed in our previous study, and thousands of lncRNAs and mRNAs were differentially expressed between CRC tissues and NCTs (22). Subsequently, LncTar, which is an efficient tool for predicting RNA targets of IncRNAs that is provided by Cui Lab from the Department of Biomedical Informatics, Peking University Health Science Center (http://www.cuilab. cn/lnctar) (23), was used. The Gibbs chemical bond free energy between tumor protein 63 (TP63; NM_003722.4) and candidate IncRNAs was determined. Using a value of $<0.05$ as the threshold, IncRNAs below that threshold were considered to have a lower Gibbs chemical bond free energy. A total of 24 lncRNAs were predicted to target TP63; among those, six were upregulated and 18 were downregulated in a CRC lncRNA microarray. The top five upregulated TP63-associated lncRNAs are listed in Table III.

Expression of a newly identified IncRNA is upregulated in $C R C$ tissues. To further verify the IncRNAs that are associated with p63 in tumor samples, RT-qPCR was performed to assess the expression levels of upregulated lncRNAs in 10 pairs of matched CRC tissues and NCTs. Among these lncRNAs, IncRNA RP1-85F18.6 (ENST00000415054) was the most markedly increased in CRC tissues (data not shown). Therefore, lncRNA RP1-85F18.6 was selected for subsequent experiments. Compared with the NCTs, lncRNA RP1-85F18.6 expression was increased in 24 tumor tissues (70.6\%, $\mathrm{P}<0.05)$, out of the 34 pairs of matched CRC tissues and NCTs (Fig. 1A). These results provided further evidence to suggest that lncRNA RP1-85F18.6 was increased in CRC tissues. The results of RT-qPCR also demonstrated that $\triangle$ Np63 expression was increased in 22 tumor tissues $(64.8 \%$, $\mathrm{P}<0.05$ ), and TAp63 expression was reduced in 22 tumor 
Table III. Top five upregulated tumor protein 63-associated long non-coding RNAs.

\begin{tabular}{llr}
\hline Sequence name & ndG & Fold change \\
\hline ENSG00000232754IENST00000415054 & -0.0889 & 13.3513184 \\
ENSG00000257453IENST00000552367 & -0.0823 & 4.7515723 \\
ENSG00000259933|ENST00000563284 & -0.0746 & 6.7542724 \\
ENSG00000259479|ENST00000564140 & -0.0712 & 5.0080102 \\
ENSG00000204787|ENST00000447469 & -0.0698 & 10.0279087
\end{tabular}

dG, delta of Gibbs chemical bond free energy.

tissues (64.8\%, $\mathrm{P}<0.05$ ) (Fig. 1B and C). Similarly, the protein expression levels of $\triangle \mathrm{Np} 63$ were obviously increased in CRC tissues (Fig. 1D), whereas the protein expression levels of TAp63 were reduced (Fig. 1D). These results were consistent with the findings of previous studies $(13,21)$. Furthermore, lncRNA RP1-85F18.6 was positively correlated with $\triangle \mathrm{Np} 63$ expression $(r=0.678, P<0.001)$, whereas the correlation with TAp63 expression was negative $(\mathrm{r}=-0.371, \mathrm{P}=0.0308)$ (Fig. 1E and $\mathrm{F}$ ). These findings indicated that lncRNA RP1-85F18.6 may be correlated with TP63, and may facilitate the progression of CRC.

Expression of IncRNA RP1-85F18.6, $\triangle N p 63$ and TAp63 in CRC cells. To assess the expression of lncRNA RP1-85F18.6, $\triangle \mathrm{Np} 63$ and TAp63 in CRC cells, their expression was examined in various CRC cell lines (SW480, SW620 and HCT116) compared with in the NCM460 normal human colorectal epithelial cell line, using RT-qPCR and western blotting. Consistent with the results of CRC tumor samples, the mRNA expression levels of lncRNA RP1-85F18.6 and $\Delta$ Np63 were markedly upregulated, whereas those of TAp63 were downregulated in the SW480 and SW620 cell lines (Fig. 1G); the protein expression of $\triangle \mathrm{Np} 63$ and TAp63 was altered accordingly (Fig. 1H). These results further confirmed that lncRNA RP1-85F18.6 and TP63 may serve key roles in triggering the tumorigenic process in CRC. In addition, the differences in the expression of lncRNA RP1-85F18.6, $\Delta$ Np63 and TAp63 were most significant in the SW620 cell line; therefore, SW620 cells were selected for subsequent experimentation.

lncRNA RP1-85F18.6 acts as an oncogene in CRC cells. To evaluate the biological role of IncRNA RP1-85F18.6 in CRC tumorigenesis, IncRNA RP1-85F18.6 was silenced by transfecting SW620 cells with lncRNA RP1-85F18.6 siRNA or NC siRNA (Fig. 2A). The Matrigel-coated Transwell assay revealed that silencing lncRNA RP1-85F18.6 inhibited invasion of CRC cells compared with in the NC group (215 vs. 272, $\mathrm{P}<0.05$ ) (Fig. 2B). This finding suggested that lncRNA RP1-85F18.6 may promote the invasive phenotype of CRC cells. To investigate whether lncRNA RP1-85F18.6 regulated the proliferation of CRC cells, the MTT assay was performed. As shown in Fig. 2C, the proliferative ability of lncRNA RP1-85F18.6 siRNA-transfected SW620 cells was significantly reduced. Subsequently, cell cycle analysis revealed that SW620 cells exhibited a shortened $\mathrm{S}$ phase $(11.9$ vs. $22.0 \%, \mathrm{P}<0.05)$ and were arrested at the $\mathrm{G}_{2}$ phase $(17.8$ vs. $7.6 \%, \mathrm{P}<0.05)$ following transfection with lncRNA RP1-85F18.6 siRNA compared with in the NC group (Fig. 2D). Furthermore, the apoptotic rate of SW620 cells was upregulated after silencing lncRNA RP1-85F18.6 (13.38 vs. 4.81\%, P<0.05) compared with in the NC group (Fig. 2E). These results suggested that the reduced cell proliferation may be attributed to disrupted cell cycle progression and increased apoptosis.

Furthermore, $\mathrm{LDH}$ release was markedly elevated following inhibition of lncRNA RP1-85F18.6, thus indicating rupture of the plasma membrane and pyroptosis (Fig. 2F). This result suggested that the reduced proliferation may also be caused by cell pyroptosis. GSDMD is a member of the gasdermin family, which has been confirmed to be the main effector molecule for pyroptosis $(24,25)$. In various CRC cells, the expression of GSDMD was revealed to be downregulated, both at the mRNA and protein levels (Fig. 2G and H). Furthermore, it was demonstrated that, although silencing lncRNA RP1-85F18.6 did not alter the full-length protein expression of GSDMD, it increased GSDMD-N domain cleavage, as demonstrated by western blotting (Fig. 3A). Following cleavage from the full-length GSDMD, the GSDMD-N domain acts as the executioner of pyroptosis, perforating the cell membrane, and causing cell swelling and lysis (26).

lncRNA RP1-85F18.6 acts as an oncogene in CRC cells through regulating $\triangle N p 63$. To further investigate the underlying mechanisms by which lncRNA RP1-85F18.6 mediates the biological functions of CRC cells, the present study examined whether IncRNA RP1-85F18.6 regulates the expression of $\triangle \mathrm{Np} 63$ and TAp63. IncRNA RP1-85F18.6 was silenced in SW620 cells, and the RT-qPCR and western blotting results revealed that the expression of $\triangle \mathrm{Np} 63$ was reduced at both the mRNA and protein levels (Fig. 3A and B). Unexpectedly, there were no significant alterations in the expression of TAp63 at the mRNA or protein levels (Fig. 3A and B).

Subsequently, SW620 cells were transfected with lncRNA RP1-85F18.6-expressing vector, or were co-transfected with lncRNA RP1-85F18.6-expressing vector and the $\Delta$ Np63 siRNA (Fig. 3C-G), which was revealed to decrease $\Delta$ Np63 expression (Fig. 4). The expression of lncRNA RP1-85F18.6 was markedly upregulated following transfection with lncRNA RP1-85F18.6-expressing vector (Fig. 3C). Compared with the NC group, overexpression of lncRNA RP1-85F18.6 increased the percentage of cells in $\mathrm{S}$ phase and decreased the percentage 


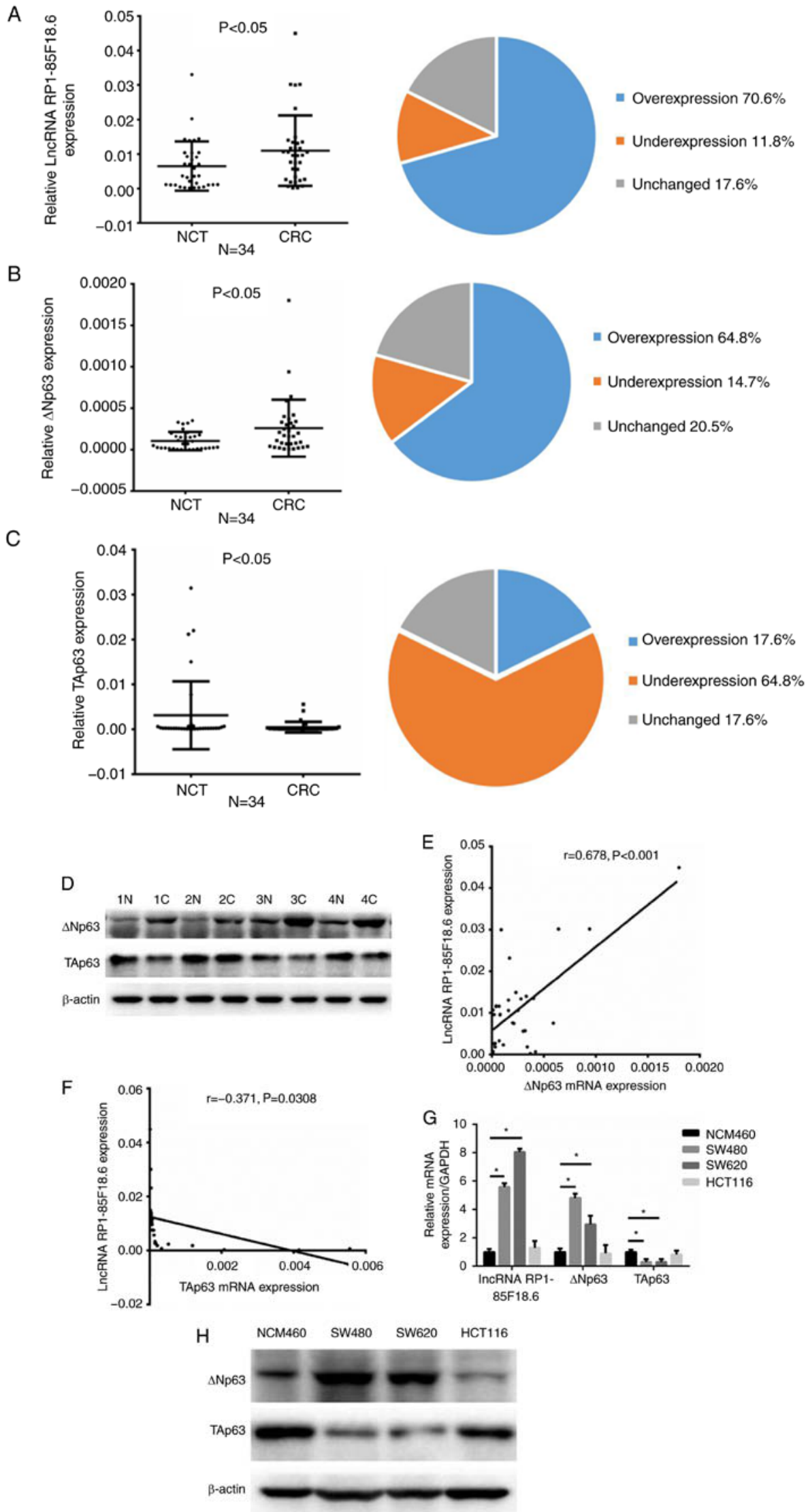

Figure 1. Expression of 1ncRNA RP1-85F18.6, $\Delta$ Np63 and TAp63 in CRC tissues and cell lines. (A-C) Expression of 1ncRNA RP1-85F18.6, $\Delta$ Np63 and TAp63 in CRC tissues, as assessed by RT-qPCR $(n=34, \mathrm{P}<0.05)$. The mean expression levels are shown in the left scatter diagram and the expression distributions are summarized in the right pie chart. GAPDH was used for normalization. (D) Representative results of increased $\Delta$ Np63 expression and reduced TAp63 expression in CRC tissues, as assessed by western blotting (N, NCT; C, CRC). (E and F) lncRNA RP1-85F18.6 was positively correlated with $\Delta$ Np63 and negatively correlated with TAp63 in CRC samples. (G and H) Expression levels of lncRNA RP1-85F18.6, $\Delta$ Np63 and TAp63 in various cell lines were evaluated by RT-qPCR and western blotting; $\mathrm{n}=3$, ${ }^{*} \mathrm{P}<0.05$. CRC, colorectal cancer; lncRNA, long non-coding RNA; NCT, non-cancerous tissue; RT-qPCR, reverse transcription-quantitative polymerase chain reaction. 
$A \stackrel{\infty}{\stackrel{\infty}{\infty}}$

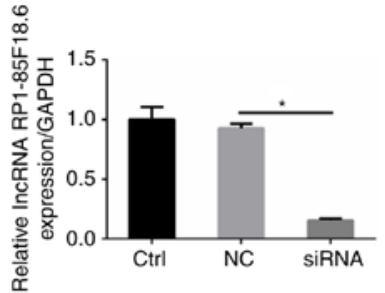

B
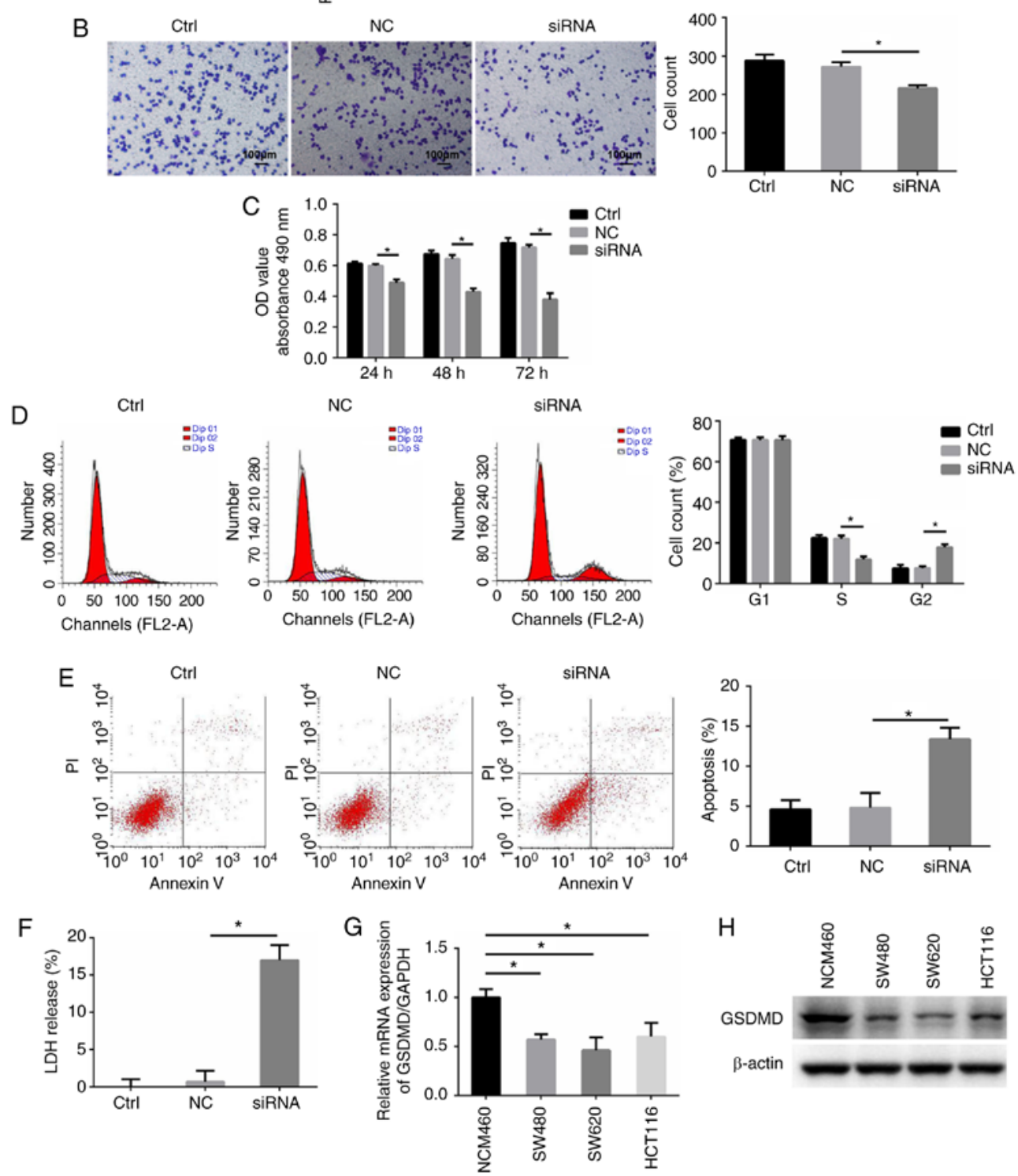

Figure 2. Biological functions of lncRNA RP1-85F18.6 in CRC cells. The CRC cells were transfected with lncRNA RP1-85F18.6 siRNA or NC siRNA. (A) Post-transfection with lncRNA RP1-85F18.6 siRNA, the expression levels of 1ncRNA RP1-85F18.6 were assessed by RT-qPCR. (B) Matrigel-coated Transwell assay was used to determine the invasive ability of cells; magnification, x200. (C) Proliferative ability was assessed by the MTT assay. (D and E) Cell cycle distribution and apoptosis were determined by flow cytometric analysis. (F) LDH release was measured by colorimetric assay. (G and $\mathrm{H}$ ) mRNA and protein expression levels of GSDMD in various cell lines were evaluated by RT-qPCR and western blotting, respectively. $n=3$, ${ }^{*} \mathrm{P}<0.05$. CRC, colorectal cancer; GSDMD, gasdermin D; LDH, lactate dehydrogenase; lncRNA, long non-coding RNA; NC, negative control; RT-qPCR, reverse transcription-quantitative polymerase chain reaction; siRNA, small interfering RNA.

of cells in $\mathrm{G}_{2}$ phase (Fig. 3D). In addition, increased lncRNA RP1-85F18.6 expression promoted cell proliferation (Fig. 3E) and invasion (Fig. 3F), and inhibited apoptosis (Fig. 3G) and cleavage of the GSDMD-N domain (Fig. $3 \mathrm{H}$ ) in SW620 cells. However, co-transfection with lncRNA RP1-85F18.6-expressing vector and $\triangle \mathrm{Np} 63$ siRNA reversed these tumor-promoting effects. These data suggested that IncRNA RP1-85F18.6 may induce the proliferation, invasion and cell cycle disruption, and inhibit apoptosis and pyroptosis of $\mathrm{CRC}$ cells through regulating $\triangle \mathrm{Np} 63$.

Association of IncRNA RP1-85F18.6, $\triangle N p 63$ and GSDMD expression with clinicopathological parameters. To establish the association of GSDMD with CRC, the mRNA and protein expression levels of GSDMD were detected in CRC tissues. The mRNA expression levels of GSDMD 

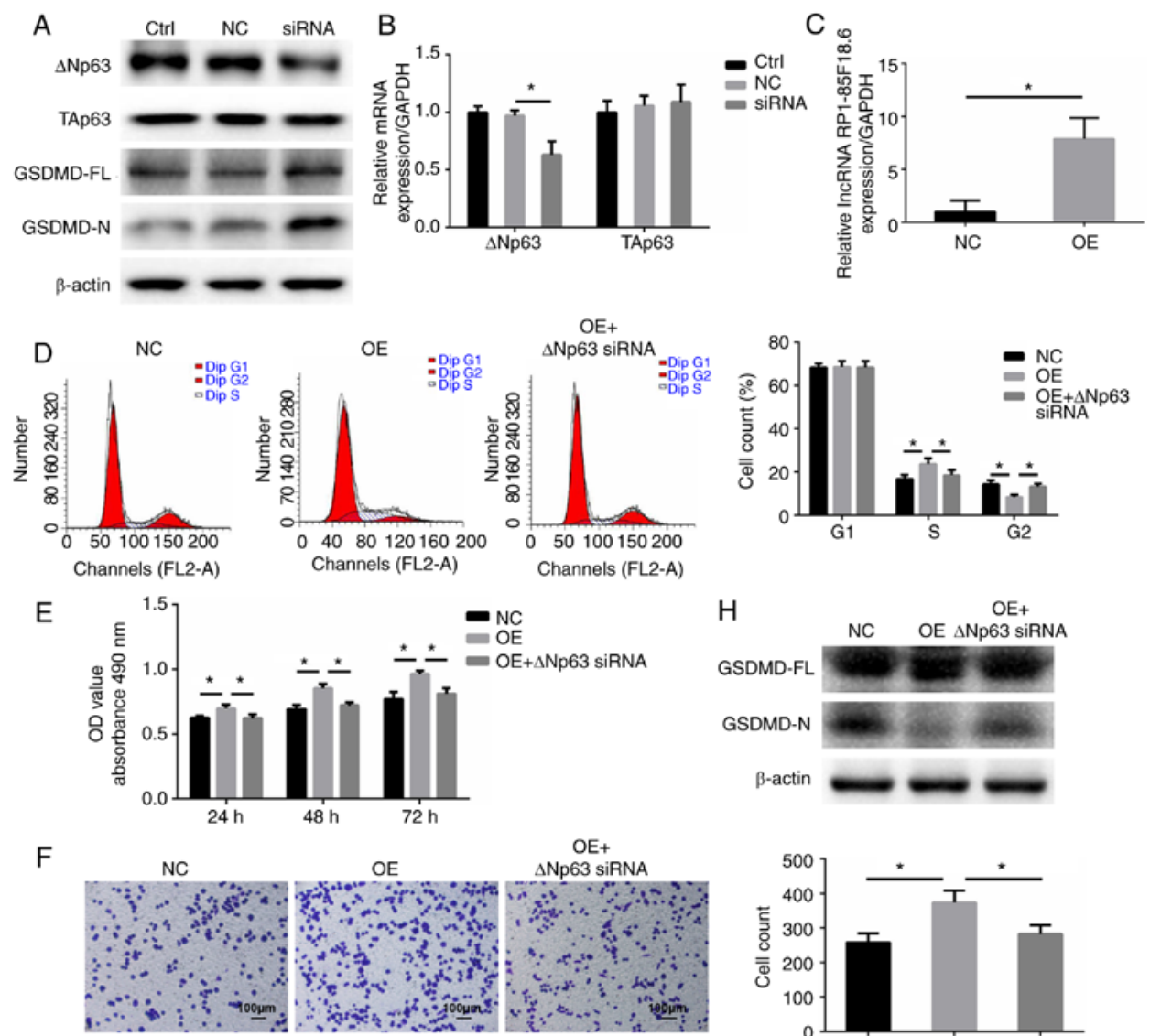

$\mathrm{OE}+$
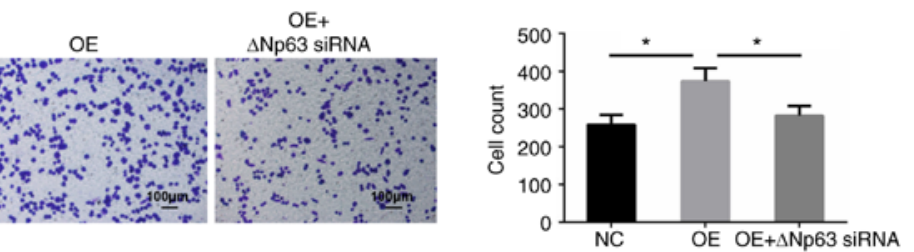

G

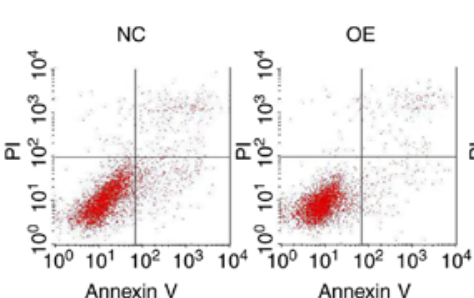

OE+
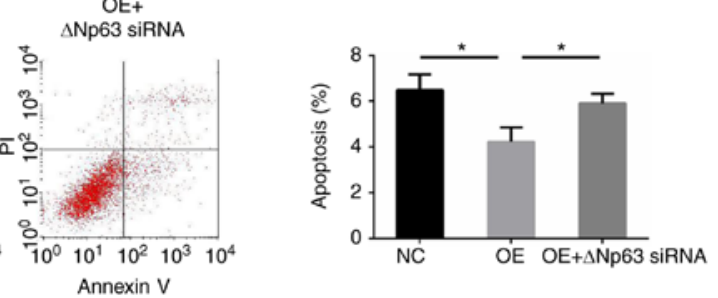

Figure 3. IncRNA RP1-85F18.6 mediates biological functions of CRC cells through regulating $\Delta$ Np63. (A) Western blotting was performed to evaluate $\Delta$ Np63, TAp63, GSDMD-FL and GSDMD-N expression after knockdown of lncRNA RP1-85F18.6. (B) Following transfection with lncRNA RP1-85F18.6 siRNA, the expression levels of $\triangle \mathrm{Np} 63$ and TAp63 were assessed by RT-qPCR. CRC cells were transfected with empty vector or lncRNA RP1-85F18.6-expressing vector, or were co-transfected with lncRNA RP1-85F18.6-expressing vector and $\Delta$ Np63 siRNA. (C) Following transfection with the lncRNA RP1-85F18.6-expressing vector, the expression of lncRNA RP1-85F18.6 was assessed by RT-qPCR. (D) Cell cycle distribution was determined by flow cytometry. (E) Proliferative ability of CRC cells was evaluated by the MTT assay. (F) Matrigel-coated Transwell assay was used to determine invasive ability of cells; magnification, x200. (G) Flow cytometric analysis was applied to evaluate cell apoptosis. (H) Protein expression levels of GSDMD-FL and GSDMD-N were determined by western blotting. $\mathrm{n}=3$, "P<0.05. CRC, colorectal cancer; GSDMD, gasdermin D; GSDMD-FL, GSDMD-full length; GSDMD-N, GSDMD-N domain; lncRNA, long non-coding RNA; NC, negative control; OD, optical density; OE, overexpression of lncRNA RP1-85F18.6; RT-qPCR, reverse transcription-quantitative polymerase chain reaction; siRNA, small interfering RNA.

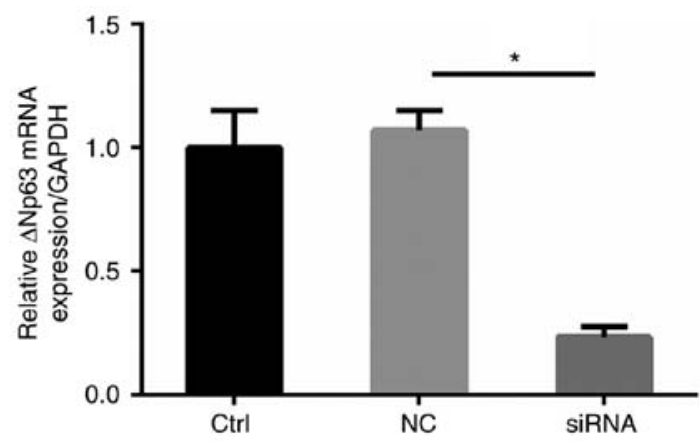

Figure 4. $\Delta$ Np63 expression was knocked down by siRNA in colorectal cancer cells. mRNA expression levels were determined using reverse transcription-quantitative polymerase chain reaction. $n=3,{ }^{*} \mathrm{P}<0.05$. NC, negative control; siRNA, small interfering RNA. were reduced in 26 tumor tissues $(76.4 \%, \mathrm{P}<0.05)$ (Fig. 5A). Consistently, the protein expression levels of GSDMD were markedly decreased in CRC tissues (Fig. 5B). Furthermore, the association of 1ncRNA RP1-85F18.6, $\Delta$ Np63 and GSDMD expression with the clinicopathological characteristics of patients with CRC was evaluated. As shown in Table I, lncRNA RP1-85F18.6 and $\Delta$ Np63 expression was positively correlated with lymph node and distant metastases $(\mathrm{P}<0.05)$. Conversely, an inverse association was determined between GSDMD expression and lymph node and distant metastases $(\mathrm{P}<0.05)$. In addition, there were no statistically significant associations between lncRNA RP1-85F18.6, $\triangle \mathrm{Np} 63$ or GSDMD expression, and other clinicopathological parameters $(\mathrm{P}>0.05)$. 

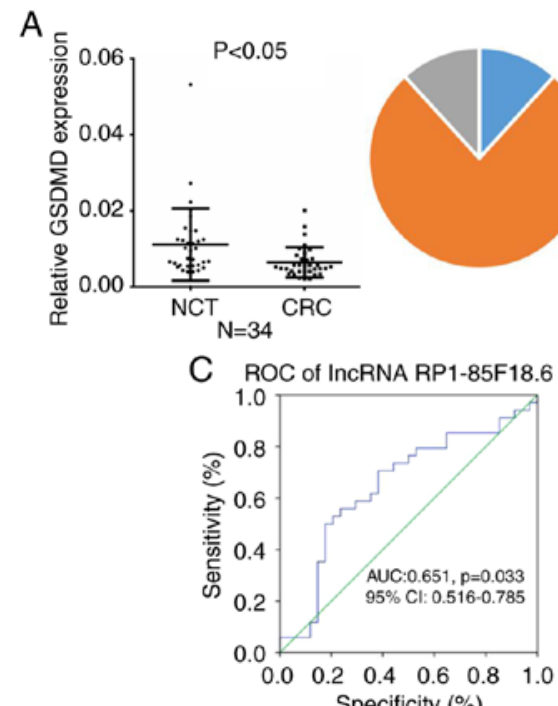

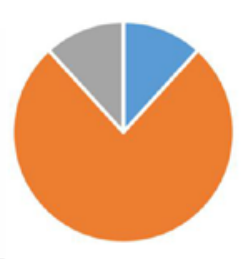

= Overexpression $11.8 \%$

= Underexpression $76.4 \%$

- Unchanged $11.8 \%$
B

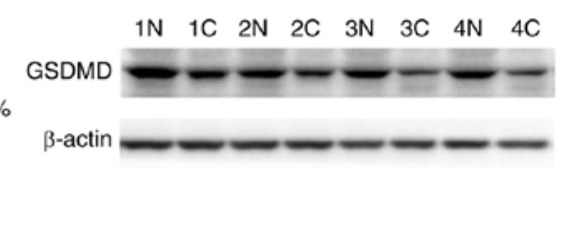

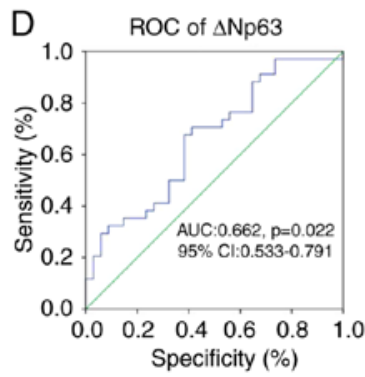

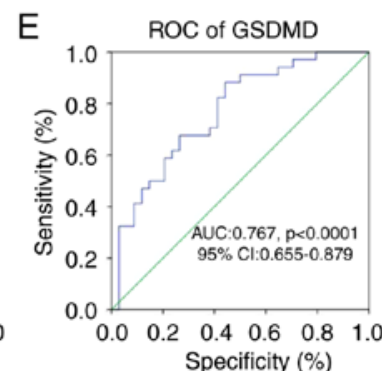

Figure 5. Association between lncRNA RP1-85F18.6, $\triangle \mathrm{Np} 63$ and GSDMD expression, and clinicopathological characteristics of patients with CRC. (A) Expression of GSDMD in CRC tissues, as assessed by reverse transcription-quantitative polymerase chain reaction ( $\mathrm{n}=34, \mathrm{P}<0.05)$. The mean expression of GSDMD is shown in the left scatter diagram and the expression distribution is summarized in the right pie chart. GAPDH was used for normalization. (B) Representative results of reduced expression of GSDMD in CRC tissues from four cases, as assessed by western blotting (N, NCT; C, CRC). (C-E) ROC curve analysis of the expression levels of lncRNA RP1-85F18.6, $\triangle \mathrm{Np} 63$ and GSDMD for discriminating CRC from NCT samples. AUC, area under the ROC curve; CRC, colorectal cancer; GSDMD, gasdermin D; IncRNA, long non-coding RNA; NCT, non-cancerous tissue; ROC, receiver operating characteristic.

The present study also demonstrated that lncRNA RP1-85F18.6, $\triangle \mathrm{Np} 63$ and GSDMD were suitable predictors of CRC through a ROC curve analysis. The AUC of lncRNA RP1-85F18.6 for CRC was 0.651 [95\% confidence interval (CI): $0.516-0.785 ; \mathrm{P}=0.033$ ], with $55.9 \%$ sensitivity and $76.5 \%$ specificity (Fig. 5C). The AUC of $\triangle \mathrm{Np} 63$ for CRC was 0.662 [95\% CI: $0.533-0.791 ; \mathrm{P}=0.022$ ], with $70.6 \%$ sensitivity and $58.8 \%$ specificity (Fig. 5D). The AUC of GSDMD for CRC was 0.767 [95\% CI: 0.655-0.879; $\mathrm{P}<0.0001$ ], with $88.2 \%$ sensitivity and $55.9 \%$ specificity (Fig. $5 \mathrm{E}$ ). These results suggested that lncRNA RP1-85F18.6, $\Delta$ Np63 and GSDMD may be implicated in CRC tumorigenesis and metastasis, and may serve as potential prognostic biomarkers of CRC in the future.

\section{Discussion}

Initially, lncRNAs were considered to be 'transcriptional noise'; however, over the past few years, research has indicated that IncRNAs may act as oncogenes or tumor suppressor genes, and exert a multitude of biological effects on various types of cancer, including CRC $(20,27,28)$. Accumulating evidence has indicated that IncRNAs regulate the proliferation, migration, invasion, apoptosis, pyroptosis and metastasis of cancer cells during tumorigenesis $(6,7)$.

In the present study, the expression levels of the newly identified IncRNA RP1-85F18.6 were upregulated in CRC tissues and cell lines, thus indicating that it may have a key role in CRC tumorigenesis. Subsequently, the biological role of lncRNA RP1-85F18.6 was evaluated in CRC cells, and the underlying molecular mechanisms were explored. The results demonstrated that lncRNA RP1-85F18.6 triggered the proliferation, invasion and cell cycle disruption, and suppressed apoptosis and pyroptosis of CRC cells. In addition, it was revealed that knockdown of lncRNA RP1-85F18.6 decreased $\Delta$ Np63 expression without affecting TAp63 expression. Furthermore, the results demonstrated that the tumor-promoting effects of 1ncRNA RP1-85F18.6 overexpression were reversed by knockdown of $\Delta \mathrm{Np} 63$. It is well known that $\mathrm{p} 53$ serves as a tumor suppressor in CRC cells through the regulation of various target genes (29). $\Delta \mathrm{Np} 63$, which is a member of the p53 family, acts as an oncogene (30,31); specifically, $\Delta \mathrm{Np} 63$ acts as a transcriptional repressor and oncoprotein through opposing the activity of p53 $(32,33)$. Previous studies have reported that $\Delta \mathrm{Np} 63$ facilitates transition through the cell cycle, proliferation, migration, invasion and metastasis of cancer cells, whereas it inhibits apoptosis, through stimulating the expression of several target genes $(12,13,34)$. These biological functions of $\Delta \mathrm{Np} 63$ are in accordance with those of lncRNA RP1-85F18.6, which further supports the present evidence. These findings indicated that the tumor-promoting effects of lncRNA RP1-85F18.6 on CRC cells are partially mediated through the $\Delta$ Np63 signaling pathway.

Based on the results obtained over the last few years, lncRNAs appear to regulate gene expression at epigenetic, transcriptional or post-transcriptional levels (5). In the present study, knockdown of lncRNA RP1-85F18.6 reduced $\Delta$ Np63 expression at both the mRNA and protein levels, thus suggesting that lncRNA RP1-85F18.6 regulates $\Delta$ Np63 partly through transcriptional regulation. Previous research revealed that $\operatorname{lncRNA}$ PURPL regulates $\mathrm{p} 53$ expression and stability through association with MYB Binding Protein 1a, a protein that binds to and stabilizes p53 (16). It may be hypothesized that lncRNA RP1-85F18.6 regulates $\Delta$ Np63 by a certain gene that promotes $\Delta \mathrm{Np} 63$ stability; we hope to elucidate this in future studies.

In the present study, silencing lncRNA RP1-85F18.6 also induced pyroptosis of CRC cells through cleavage of GSDMD. To the best of our knowledge, there are few studies focusing on the role of non-protein-coding genes in pyroptosis (7). In 
addition to apoptosis, pyroptosis is another type of programmed cell death, which is characterized by plasma membrane rupture. Recently, Shi et al redefined pyroptosis as gasdermin-mediated programmed necrosis (26). Furthermore, Wang et al revealed that chemotherapeutic drugs induce pyroptosis of cancer cells through cleavage of gasdermin E (35). In the present study, cleavage of GSDMD, which was induced by silencing lncRNA RP1-85F18.6, promoted the pyroptosis of CRC cells, thus suggesting that GSDMD may represent a novel focus in CRC research.

Carcinoembryonic antigen (CEA) is the most widely used molecular marker of CRC, which has been proven to be a valuable tool for the diagnosis of CRC (36). Previous research demonstrated that the sensitivity of CEA is $46.59 \%$ and its specificity is $80 \%$ (37). In the present study, the sensitivity and specificity values of lncRNA RP1-85F18.6 were 55.9 and $76.5 \%$, respectively, which are comparable with those of CEA. These findings suggested that lncRNA RP1-85F18.6 may be a valuable prognostic and diagnostic biomarker for $\mathrm{CRC}$ in the future.

In conclusion, to the best of our knowledge, the present study is the first to report that lncRNA RP1-85F18.6 expression may be increased in CRC tissues and cell lines. This lncRNA was revealed to promote proliferation, invasion and cell cycle disruption, whereas it inhibited the apoptosis and pyroptosis of CRC cells through inducing $\triangle \mathrm{Np} 63$. In addition, the present findings indicated that lncRNA RP1-85F18.6, $\Delta$ Np63 and GSDMD may prove to be valuable prognostic and diagnostic biomarkers for early-stage CRC in the future.

\section{Acknowledgements}

Not applicable.

\section{Funding}

The present study was supported by the New Xiangya Talent Project of The Third Xiangya Hospital of Central South Univ ersity (grant no. JY201508).

\section{Availability of data and materials}

All data generated or analyzed during this study are included in this published article.

\section{Author's contributions}

YM performed the experiments and wrote the manuscript; YC statistically analyzed the data; CL collected the tissues and analyzed the clinical characteristics of all patients; $\mathrm{GH}$ designed the experiments, analyzed the ata, supervised the experiments and gave final approval of the version to be published. All authors read and approved the final manuscript.

\section{Ethics approval and consent to participate}

The present study was approved by the Ethics Committee of The Third Xiangya Hospital of Central South University (No. 2014-S009), and patients provided written informed consent.

\section{Patient consent for publication}

The patients provided consent for publication.

\section{Competing interests}

The authors declare that they have no competing interests.

\section{References}

1. Siegel RL, Miller KD, Fedewa SA, Ahnen DJ, Meester RG, Barzi A and Jemal A: Colorectal cancer statistics, 2017. CA Cancer J Clin 67: 177-193, 2017.

2. Lozano R, Naghavi M, Foreman K, Lim S, Shibuya K, Aboyans V, Abraham J, Adair T, Aggarwal R, Ahn SY, et al: Global and regional mortality from 235 causes of death for 20 age groups in 1990 and 2010: A systematic analysis for the Global burden of disease study 2010. Lancet 380: 2095-2128, 2012.

3. Siegel R, Desantis C and Jemal A: Colorectal cancer statistics, 2014. CA Cancer J Clin 64: 104-117, 2014.

4. Esteller M: Non-coding RNAs in human disease. Nat Rev Genet 12: 861-874, 2011

5. Yang Y, Junjie P, Sanjun C and Ma Y: Long non-coding RNAs in colorectal cancer: Progression and future directions. J Cancer 8: 3212-3225, 2017.

6. Deng H, Wang JM, Li M, Tang R, Tang K, Su Y, Hou Y and Zhang J: Long non-coding RNAs: New biomarkers for prognosis and diagnosis of colon cancer. Tumour Biol 39: $1010428317706332,2017$.

7. Zhang Y, Liu X, Bai X, Lin Y, Li Z, Fu J, Li M, Zhao T, Yang H, $\mathrm{Xu}$, et al: Melatonin prevents endothelial cell pyroptosis via regulation of long noncoding RNA MEG3/miR-223/NLRP3 axis. J Pineal Res 64: doi: 10.1111/jpi.12449, 2018.

8. Chen Y, Peng Y, Fan S, Li Y, Xiao ZX and Li C: A double dealing tale of p63: An oncogene or a tumor suppressor. Cell Mol Life Sci 75: 965-973, 2018.

9. Gressner O, Schilling T, Lorenz K, Schulze Schleithoff E, Koch A, Schulze-Bergkamen H, Lena AM, Candi E, Terrinoni A, Catani MV, et al: TAp63alpha induces apoptosis by activating signaling via death receptors and mitochondria. EMBO J 24: 2458-2471, 2005.

10. Wu G, Nomoto S, Hoque MO, Dracheva T, Osada M, Lee CC, Dong SM, Guo Z, Benoit N, Cohen Y, et al: DeltaNp63alpha and TAp63alpha regulate transcription of genes with distinct biological functions in cancer and development. Cancer Res 63: 2351-2357, 2003.

11. Srivastava K, Pickard A, McDade S and McCance DJ: p63 drives invasion in keratinocytes expressing HPV16 E6/E7 genes through regulation of Src-FAK signalling. Oncotarget 8: 16202-16219, 2017.

12. He YF, Tian DY, Yi ZJ, Yin ZK, Luo CL, Tang W and Wu XH: Upregulation of cell adhesion through delta Np63 silencing in human 5637 bladder cancer cells. Asian J Androl 14: 788-792, 2012.

13. Compagnone M, Gatti V, Presutti D, Ruberti G, Fierro C, Markert EK, Vousden KH, Zhou H, Mauriello A, Anemone L, et al: $\Delta \mathrm{Np} 63$-mediated regulation of hyaluronic acid metabolism and signaling supports HNSCC tumorigenesis. Proc Natl Acad Sci USA 114: 13254-13259, 2017.

14. Li H, Jiang $X$ and Niu X: Long non-coding RNA reprogramming (ROR) promotes cell proliferation in colorectal cancer via affecting P53. Med Sci Monit 23: 919-928, 2017.

15. Thorenoor N, Faltejskova-Vychytilova P, Hombach S, Mlcochova J, Kretz M, Svoboda M and Slaby O: Long non-coding RNA ZFAS1 interacts with CDK1 and is involved in p53-dependent cell cycle control and apoptosis in colorectal cancer. Oncotarget 7: 622-637, 2016.

16. Li XL, Subramanian M, Jones MF, Chaudhary R, Singh DK, Zong X, Gryder B, Sindri S, Mo M, Schetter A, et al: Long noncoding RNA PURPL suppresses basal p53 levels and promotes tumorigenicity in colorectal cancer. Cell Rep 20: 2408-2423, 2017.

17. Edge SB and Compton CC: The American Joint Committee on cancer: The 7th edition of the AJCC cancer staging manual and the future of TNM. Ann Surg Oncol 17: 1471-1474, 2010.

18. Livak KJ and Schmittgen TD: Analysis of relative gene expression data using real-time quantitative PCR and the $2^{-\Delta \Delta C_{\mathrm{T}}}$ method. Methods 25: 402-408, 2001. 
19. Yun DP, Wang YQ, Meng DL, Ji YY, Chen JX, Chen HY and Lu DR: Actin-capping protein CapG is associated with prognosis, proliferation and metastasis in human glioma. Oncol Rep 39: 1011-1022, 2018.

20. Xie S, Ge Q, Wang X, Sun X and Kang Y: Long non-coding RNA ZFAS1 sponges miR-484 to promote cell proliferation and invasion in colorectal cancer. Cell Cycle 17: 154-161, 2018.

21. Chen Y, Zhang Y, He J, Fu Y, Lin C and Li X: MicroRNA-133b is regulated by TAp63 while no gene mutation is present in colorectal cancer. Oncol Rep 37: 1646-1652, 2017.

22. Wu H, Wu R, Chen M, Li D, Dai J, Zhang Y, Gao K, Yu J, Hu G Guo Y, et al: Comprehensive analysis of differentially expressed profiles of lncRNAs and construction of miR-133b mediated ceRNA network in colorectal cancer. Oncotarget 8: 21095-21105, 2017.

23. Li J, Ma W, Zeng P, Wang J, Geng B, Yang J and Cui Q: LncTar: A tool for predicting the RNA targets of long noncoding RNAs. Brief Bioinform 16: 806-812, 2015.

24. Shi J, Zhao Y, Wang K, Shi X, Wang Y, Huang H, Zhuang Y, Cai T, Wang F and Shao F: Cleavage of GSDMD by inflammatory caspases determines pyroptotic cell death. Nature 526: 660-665, 2015.

25. Ding J, Wang K, Liu W, She Y, Sun Q, Shi J, Sun H, Wang DC and Shao F: Pore-forming activity and structural autoinhibition of the gasdermin family. Nature 535: 111-116, 2016.

26. Shi J, Gao W and Shao F: Pyroptosis: Gasdermin-mediated programmed necrotic cell death. Trends Biochem Sci 42: 245-254, 2017.

27. Chen DL, Lu YX, Zhang JX, Wei XL, Wang F, Zeng ZL, Pan ZZ, Yuan YF, Wang FH, Pelicano $\mathrm{H}$, et al: Long non-coding RNA UICLM promotes colorectal cancer liver metastasis by acting as a ceRNA for microRNA-215 to regulate ZEB2 expression. Theranostics 7: 4836-4849, 2017.

28. Zhang J, Li Z, Liu L, Wang Q, Li S, Chen D, Hu Z, Yu T, Ding J, Li J, et al: Long noncoding RNA TSLNC8 is a tumor suppressor that inactivates the interleukin-6/STAT3 signaling pathway. Hepatology 67: 171-187, 2018.

29. Wu Q, Deng J, Fan D, Duan Z, Zhu C, Fu R and Wang S: Ginsenoside Rh4 induces apoptosis and autophagic cell death through activation of the ROS/JNK/p53 pathway in colorectal cancer cells. Biochem Pharmacol 148: 64-74, 2017.
30. Liu K, Yao H, Lei S, Xiong L, Qi H, Qian K, Liu J, Wang P and Zhao H: The miR-124-p63 feedback loop modulates colorectal cancer growth. Oncotarget 8: 29101-29115, 2017.

31. Nayak KB, Kuila N, Das Mohapatra A, Panda AK and Chakraborty S: EVI1 targets $\Delta \mathrm{Np} 63$ and upregulates the cyclin dependent kinase inhibitor p21 independent of p53 to delay cell cycle progression and cell proliferation in colon cancer cells. Int J Biochem Cell Biol 45: 1568-1576, 2013.

32. Balboni AL, Cherukuri P, Ung M, DeCastro AJ, Cheng $\mathrm{C}$ and DiRenzo J: p53 and $\Delta$ Np63 $\alpha$ coregulate the transcriptional and cellular response to TGF $\beta$ and BMP signals. Mol Cancer Res 13: 732-742, 2015.

33. He Y, Wu X, Tang W, Tian D, Luo C, Yin Z and Du H: Impaired delta NP63 expression is associated with poor tumor development in transitional cell carcinoma of the bladder. J Korean Med Sci 23: 825-832, 2008

34. Giacobbe A, Compagnone M, Bongiorno-Borbone L, Antonov A, Markert EK, Zhou JH, Annicchiarico-Petruzzelli M, Melino G and Peschiaroli A: p63 controls cell migration and invasion by transcriptional regulation of MTSS1. Oncogene 35: 1602-1608, 2016.

35. Wang Y, Gao W, Shi X, Ding J, Liu W, He H, Wang K and Shao F: Chemotherapy drugs induce pyroptosis through caspase-3 cleavage of a gasdermin. Nature 547: 99-103, 2017.

36. Thomas DS, Fourkala EO, Apostolidou S, Gunu R, Ryan A Jacobs I, Menon U, Alderton W, Gentry-Maharaj A and Timms JF: Evaluation of serum CEA, CYFRA21-1 and CA125 for the early detection of colorectal cancer using longitudinal preclinical samples. Br J Cancer 113: 268-274, 2015.

37. Gao Y, Wang J, Zhou Y, Sheng S, Qian SY and Huo X: Evaluation of serum CEA, CA19-9, CA72-4, CA125 and ferritin as diagnostic markers and factors of clinical parameters for colorectal cancer. Sci Rep 8: 2732, 2018.

This work is licensed under a Creative Commons Attribution-NonCommercial-NoDerivatives 4.0 International (CC BY-NC-ND 4.0) License. 